\title{
Uso potencial dos anticonvulsivantes no tratamento ambulatorial da dependência de álcool
}

\author{
Potential use of the anticonvulsants in the outpatient treatment of alcohol \\ dependence
}

\author{
Luís André Castro e Carla Couzi
}

\section{Resumo}

Atualmente três medicações (dissulfiram, naltrexona e acamprosato) são aprovadas pela Food and Drug Administration (FDA) para tratar a dependência de álcool. As drogas anticonvulsivantes clássicas são raramente empregadas como alternativa por causa dos seus efeitos colaterais, mas a sua última geração pode ser útil. Os anticonvulsivantes podem ser uma alternativa aos benzodiazepínicos (BZD) e a outros tratamentos farmacológicos na prevenção de complicações na desintoxicação por apresentarem ausência de propriedades aditivas e um melhor perfil de efeitos adversos do que os anticonvulsivantes clássicos. Anticonvulsivantes como carbamazepina, ácido valpróico, gabapentina e topiramato demonstraram-se excelentes tratamentos para síndrome de abstinência do álcool e prevenção de recaídas. Embora nenhum desses agentes tenha sido aprovado pela FDA, existe uma crescente evidência na literatura que apóia o seu uso.

Palavras-chave: anticonvulsivantes, alcoolismo, carbamazepina, ácido valpróico.

\begin{abstract}
Currently three medications (disulfiram, naltrexone and acamprosate) are approved by the FDA to treat alcohol dependence by the FDA. The classical anticonvulsive drugs are rarely employed as an alternative because of their side effects, but the latest generation of anticonvulsants could be useful. The anticonvulsants can be a alternative to BZD and other pharmacological treatments in the prevention of complications during the detoxification therapy, because of the absence of addictive properties and a better adverse effects profile than classical anticonvulsant drugs. Anticonvulsants such as carbamazepine, valproic acid, gabapentin and topiramate have shown to be excellent treatment for alcohol withdrawal and for the prevention of alcohol relapse. Although none of these agents have been approved by the FDA yet, there is growing evidence in the literature to support their use.

Key words: anticonvulsants, alcoholism, carbamazepine, valproic acid.
\end{abstract}

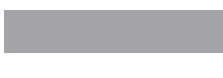

Departamento de Psiquiatria da Escola Paulista de Medicina da Universidade Federal de São Paulo (EPM/UNIFESP) (Castro LA)

Recebido

07-03-06

Aprovado

16-08-06

Correspondência para: Luís André Castro

Rua Botucatu, 394 - Vila Clementino - 04023-061 - São Paulo-SP - Tel.: (11) 5575-1708

e-mail: castro@psiquiatria.epm.br
} 


\section{Introdução}

Os avanços da neurociência têm impulsionado o desenvolvimento do interesse clínico e científico na descoberta de intervenções farmacológicas (IF) para tratar a síndrome de dependência do álcool (SDA), um sério problema de saúde pública que acomete cerca de $11,2 \%$ da população adulta brasileira (Carlini et al., 2002).

$\mathrm{Na}$ psiquiatria, os anticonvulsivantes (ATC) possuem um espectro de ação amplo que inclui bulimia nervosa (BN), transtornos da compulsão alimentar periódica (TCAP) (Appolinario-Bacaltchuk, 2002), do pânico, de estresse pós-traumático (Litten et al., 2005), de controle dos impulsos (Zullino et al., 2004) e os casos refratários de transtorno afetivo bipolar (TAB) (Moreno et al., 2004).

Entre as razões que tornam os ATCs uma classe de IF promissora na prevenção de recaídas no tratamento da SDA destacam-se os efeitos anticonvulsivante (Malcolm et al., 2001) e anti-kindling (Goddard et al., 1969; Ballenger-Post, 1978; Lechtenberg-Worner, 1991; Moak-Anton, 1996; McNamara-Wada, 1997; Becker, 1998), a ausência de potencial de abuso, a ação estabilizadora do humor no caso de co-morbidades psiquiátricas com $\mathrm{TAB}$, a menor interação com o álcool, quando em comparação com os benzodiazepínicos (BDZs), o efeito anti-craving (Johnson et al., 2003) e o uso potencial no tratamento da síndrome de abstinência protraída (SAP). Há que se ressaltar a baixa incidência de efeitos adversos, como, por exemplo, déficits cognitivos, o que implica uma melhor tolerabilidade (Johnson et al., 2005).

Os principais objetivos desta revisão de literatura são atualizar os conhecimentos acerca da eficácia terapêutica e do potencial uso dos ATCs no tratamento da SDA, bem como propor alternativas aos tratamentos convencionais. Neste artigo priorizaremos a carbamazepina (CBZ), o valproato de sódio, o topiramato (TPM) e a gabapentina, em decorrência da disponibilidade de ensaios clínicos controlados na literatura especializada.

\section{Intervenções farmacológicas preconizadas na SDA}

\section{Dissulfiram}

O dissulfiram (DSF) é um inibidor da aldeído desidrogenase que causa efeitos aversivos quando o álcool é consumido. Os resultados com esse medicamento são inconsistentes na SDA (Chick et al., 1992; Carrol et al., 1998). Supõe-se que indivíduos mais idosos e socialmente estáveis tratados com DSF bebem menor número de dias que aqueles tratados com placebo (Fuller et al., 1986). O seu uso deve ser supervisionado e empregado como parte de um amplo programa de tratamento. Não há boas evidências a favor do uso de implantes de DSF, mas a possibilidade de uma injeção de depósito é intrigante. A primeira dose nunca deve ser dada até que o paciente esteja abstinente do álcool por pelo menos 12 horas. Entre seus efeitos colaterais destacam-se rash cutâneo, gosto metálico na boca, náuseas, diarréia, dor de cabeça e impotência. Pode também causar hepatite colestática e hepatite fulminante, por isso, para alguns autores, essa medicação é proscrita a pacientes com hepatite aguda, cirrose hepática com ou sem hipertensão porta e insuficiência hepática (Chick et al.,1992; Fuller-Gordis, 2004).

\section{Naltrexona}

Estudos americanos têm avaliado a eficácia do uso de naltrexona (NTX) associado com psicoterapia. A NTX é um antagonista opióide que bloqueia receptores $\mu$, $\delta$ e k. Em 1994 essa medicação foi aprovada pelo Food and Drug Administration (FDA). A maioria dos estudos tem produzido resultados positivos (Volpicelli et al., 1992; Anton et al., 1996; Monterosso et al., 2001). Entre esses, a redução do craving ou nas propriedades de reforço do álcool tem sido postulada como um possível mecanismo de ação dessa droga (O'Malley et al., 1992; Volpiceli et al., 1992).

Segundo Garbutt et al. (1999), a NTX, quando em comparação com o placebo, reduz o risco de recaída, mas não aumenta substancialmente a abstinência. As principais contraindicações ao uso da NTX são hepatite aguda, cirrose hepática (com ou sem hipertensão porta) e insuficiência hepática. 0 uso concomitante de opióides pode precipitar síndrome de abstinência, que se instala 5 minutos após a administração da medicação e dura aproximadamente 48 horas. Nesses pacientes é necessário um período mínimo de sete dias de abstinência antes de se prescrever a naltrexona. Para aqueles tratados com metadona, recomenda-se um período de abstinência maior: 10 a 14 dias (Castro e Baltieri, 2004).

\section{Acamprosato}

O acamprosato (ACP) é amplamente utilizado nos países europeus, tendo sido recentemente aprovado pela FDA. O ACP tem estrutura semelhante à do ácido gama-aminobutírico (GABA) (Litten et al., 1996), sendo também análoga ao ácido L-glutâmico (Knopfel et al., 1987), o que justifica seus mecanismos de ação envolvendo os sistemas GABAérgico e glutamatérgico, respectivamente. Estudos sugerem que sua eficácia se deva ao antagonismo de neurotransmissão do receptor N-metil-D-aspartato (NMDA) no sítio da glicina (Zeise et al., 1993).

A diminuição do consumo de álcool talvez esteja associada à redução de sintomas da síndrome de abstinência do álcool (SAA), uma vez que uma hiperexcitação do sistema nervoso central (SNC) acompanha e, presumivelmente, causa a síndrome de retirada.

Estudos em modelos animais mostram redução de sinais autonômicos e até convulsões em ratos (Spanagel et al., 1996). Parece que 0 alívio da SAP também poderia ser outro fator redutor de recaídas. Nalpas et al. (1990) sugerem que não há evidências de interação entre ACP e álcool e que, além disso, essa medicação não possui qualquer efeito sedativo-ansiolítico ou potencial de abuso. Portanto sugere-se, por exclusão, que o ACP possa reduzir o craving para o álcool ao diminuir o reforço negativo associado com a SAA (Littleton, 1995). Os principais efeitos colaterais são diarréia e cefaléia. O ACP é contra-indicado durante gravidez, amamentação e insuficiências renal e hepática.

\section{Uso potencial dos anticonvulsivantes}

\section{Síndrome de abstinência do álcool}

A administração crônica de álcool induz alterações de neuroadaptação do SNC conhecidas como down-regulation (dessensibilização ou subsensibilidade) e up-regulation (supersensibilidade ou hipersensibilidade). Esses fenômenos neuroquímicos são responsáveis pela manifestação clínica 
dos sintomas da SAA. No sistema GABAérgico, o fenômeno de down-regulation dos receptores $\mathrm{GABA}_{\mathrm{A}}$ está associado à redução do influxo de íons $\mathrm{Cl}^{-}$e, conseqüentemente, a uma diminuição da ação inibitória desse sistema sob a forma de ansiedade e excitabilidade do SNC e do sistema periférico. Conclui-se que há uma hipoatividade GABAérgica do tipo funcional. Essa hipoatividade é funcional porquanto não há evidências de alteração no número de receptores $\mathrm{GABA}_{\mathrm{A}}$ durante a exposição crônica ao álcool. Supõe-se que há redução da densidade de mRNA relacionada ao local de ação dos BDZs e do etanol. No sistema glutamatérgico o álcool atua como um antagonista dos receptores NMDA. Logo, a administração crônica do álcool cursará com o fenômeno de up-regulation, que provocará aumento da densidade desses receptores.

Em estudos com animais foram evidenciadas crises convulsivas nas primeiras 36 horas após a retirada do etanol. Estudos pré-clínicos correlacionam esse efeito neurotóxico com a hiperatividade glutamatérgica. $\mathrm{O}$ uso potencial dos anticonvulsivantes se justifica devido à correção dessas alterações neuroadaptativas dos sistemas GABAérgico e glutamatérgico, quer seja aumentando ou diminuindo atividades inibitória e/ou excitatória dos mesmos (Laranjeira et al., 2000).

Estudos pré-clínicos evidenciam que os anticonvulsivantes são eficazes na redução das convulsões e de outros sintomas que compõem a SAA, além de diminuir a tolerância aos efeitos hipnóticos do etanol (Zullino et al., 2004). A interrupção da ingestão de álcool na SDA leva à ativação compensatória do SNC, que persiste por alguns dias e resulta num estado de hiperexcitabilidade. Essa se manifesta como SAA, com sintomas variando desde tremores de extremidades até convulsões e delirium tremens (DT).

Alguns pesquisadores têm sugerido que episódios repetidos de SAA podem sensibilizar os episódios posteriores. Essa hipótese resulta no fato de que a gravidade dos sintomas da SAA pode aumentar de maneira cumulativa, com sintomas mais graves após anos de abuso do álcool (Becker, 1998).

Ballenger e Post (1978) postulam que a exacerbação progressiva da SAAé a manifestação de um mecanismo chamado kindling, que inicialmente foi descrito por Goddard et al. (1969). De acordo com esse modelo, o fenômeno kindling é considerado uma forma de potencialização de longa duração, ou seja, um tipo de sensibilização comportamental.

Estudos recentes corroboram essa hipótese, demonstrando que há correlação positiva entre a ocorrência de convulsão durante a SAA e o histórico de desintoxicações prévias (Lechtenberg e Worner, 1991; Moak e Anton, 1996). Acredita-se que repetidos estímulos subconvulsivos induzam à sensibilização do cérebro, e que esses estímulos possam ser de natureza elétrica e/ou química. Para que o fenômeno kindling se desenvolva entre dependentes de álcool são necessárias exposições repetidas e intermitentes ao álcool. A principal implicação clínica é o aumento progressivo em termos de gravidade dos sintomas da SAA. A durabilidade desse fenômeno reflete provavelmente alterações em longo prazo no circuito neuronal (McNamara e Wada, 1997). O fenômeno kindling no período de abstinência sugere que até pacientes com SAA leve devam ser tratados agressivamente a fim de evitar o agravamento dos episódios posteriores (Becker, 1998).

\section{Síndrome de dependência do álcool}

A administração crônica de álcool promove alterações nos sistemas de neurotransmissores excitatórios e inibitórios, que exercem efeitos neuromoduladores na liberação de dopamina no núcleo accumbens (NA). Isso tem estimulado estudiosos a investigarem a associação GABA e glutamato com a liberação de dopamina no NA, com intenção de descobrir novas intervenções farmacológicas (IFs) (Gonzales e Jaworski, 1997; Mihic e Harris, 1997).

A dopamina é um neuromodulador que altera a sensibilidade de neurônios-alvo aos outros neurotransmissores, particularmente o glutamato. $O$ álcool, mesmo em baixas concentrações, pode aumentar a liberação de dopamina no NA. A dopamina se liga aos receptores D1, aumentando os efeitos excitatórios que resultam da interação do glutamato com um subtipo de receptor glutamato específico, o NMDA. De forma contrária, os receptores D2 inibem os efeitos induzidos pela ligação do glutamato a um outro subtipo de receptor do glutamato, o ácido alfa-amino-3-hidróxi-5-metil-4-isoxazole propiônico (AMPA). Portanto a dopamina pode facilitar e/ou inibir a neurotransmissão excitatória, dependendo do subtipo de receptor ativado (Di Chiara, 1997).

\section{Discussão}

\section{Topiramato}

O topiramato (TPM) inicialmente foi sintetizado como um potencial hipoglicemiante, ao promover a inibição da gliconeogênese através do bloqueio da enzima frutose-1,6-bisfosfatase. A estrutura química do TPM é similar à da acetazolamida, que por sua vez tem importantes propriedades anticonvulsivantes. Essa característica possibilitou a realização de novos estudos visando investigar sua eficácia como ATC (Johnson et al., 2004).

O TPM pode ser uma opção segura e efetiva em decorrência de sua ação nos sistemas GABAérgico e glutamatérgico (Johnson et al., 2004). Esse anticonvulsivante atua como um antagonista dos efeitos do álcool sobre o sistema de recompensa cerebral ao inibir a liberação de dopamina no sistema mesocorticolímbico.

Os mecanismos propostos para a indicação do TPM no tratamento da SDA compreendem:

- a redução da administração aguda de álcool ao inibir a liberação de dopamina no NA, através da potencialização da neurotransmissão GABAérgica. Essa potencialização da atividade GABAérgica é mediada pelo aumento do influxo de íons $\mathrm{Cl}^{-}$nos sítios não-BDZs localizados nos receptores GABA . O álcool, quando ingerido de forma aguda, leva à diminuição da atividade GABAérgica na área tegmentar ventral (ATV), desinibindo os neurônios dopaminérgicos dessa região, que então liberam dopamina no NA. Logo, a administração de TPM restabeleceria esse equilíbrio ao aumentar os efeitos inibitórios dos neurônios GABAérgicos sobre a liberação de dopamina no NA, além de diminuir os efeitos excitatórios dos neurônios glutamatérgicos na ATV e no NA. A principal implicação desse suposto mecanismo de ação do TPM é a redução da atividade dopaminérgica no NA. Isto é, a quantidade de dopamina essencial para a percepção das propriedades reforçadoras diminui consideravelmente quando se administra o álcool de forma aguda (Swift, 2003; Johnson et al., 2003 e 2004; Zullino et al., 2004);

- a redução da administração crônica do álcool ao inibir a atividade glutamatérgica no NA, através de sua ação antagonista 
dos receptores AMPA/cainato. O álcool, quando ingerido de forma crônica, leva a um estado de hiperexcitabilidade dos neurônios GABAérgicos da ATV, sendo uma das principais causas de hiperatividade glutamatérgica. Isso leva à diminuição da liberação de dopamina no NA, o que pode auxiliar os dependentes crônicos de álcool na abstenção ao perceberem, em intensidade menor, as propriedades reforçadoras do álcool (Johnson et al., 2004).

Johnson et al. (2003) avaliaram o uso do TPM em um ensaio clínico randomizado (ECR). Houve melhora nos seguintes desfechos clínicos: doses por dia $(p<0,05)$; doses por dia de consumo $(p<0,05)$; porcentagem de dias de consumo pesado $(p<0,05)$; porcentagem de dias de abstinência $(p<0,05)$; por fim, diminuição dos níveis da gama-glutamil transferase (GGT) $(p<0,05)$. O TPM foi efetivo em reduzir o craving tanto na dependência de álcool do tipo $A$ como na do tipo $B$.

$\mathrm{O}$ craving foi avaliado por meio da Obsessive Compulsive Drinking Scale (OCDS), indicando que o TPM reduziu o craving e levando em consideração os escores das três subescalas do OCDS, que incluíram obsessão pelo consumo de álcool $(p<0,05)$, automaticidade do consumo de álcool $(p<0,05)$ e interferência devido ao consumo de álcool $(p<0,05)$, quando em comparação com o grupo placebo.

Os efeitos adversos associados ao uso do TPM são considerados de intensidade leve a moderada, sendo os mais comuns tontura, lentificação psicomotora, parestesias e déficits de memória. Os maiores índices de efeitos adversos classificados como moderados ocorreram no início e na oitava semana de tratamento, quando se alcançou a dose máxima de $300 \mathrm{mg} / \mathrm{dia}$. Parestesias e lentificação psicomotora foram identificadas como efeitos adversos dose-dependentes, mas com tendência de remissão quando a dose é mantida por duas ou mais semanas.

Não foram relatados eventos adversos graves, como morte, por exemplo. A perda ponderal foi mais comum no grupo tratado com $\operatorname{TPM}(54,7 \%)$ em relação ao grupo placebo $(26,7 \% ; p<0,05)$. As taxas de dropout causadas pelos efeitos adversos foram maiores no grupo placebo (6,7\% vs. 4\%) (Johnson et al., 2005).

Uma estratégia com potencial para ser empregada na prática clínica são os níveis séricos do TPM. Os valores séricos aumentam até o valor de 5,02 $\pm 0,48 \mu \mathrm{g}$ na oitava semana de tratamento. Os mesmos não foram afetados por índice de massa corporal (IMC), sexo e início precoce da SDA. A porcentagem de dias de consumo pesado de álcool correlacionou-se com os níveis séricos de TPM $(p<0,05)$.

Postula-se que exista um limiar terapêutico para o TPM (300mg/dia), ou seja, uma dose acima da qual a medicação exerça o seu efeito sobre o consumo de álcool (Johnson et al., 2005).

Algumas das IFs usadas no tratamento da SDA atuam diminuindo o craving, que é considerado um bom indicador de resposta terapêutica em ensaios clínicos. Supõe-se que o craving está associado ao aumento da probabilidade de recaídas (Hartz et al., 2001). Assim sendo, conclui-se que o TPM reduz o consumo de álcool ao diminuir a intensidade e a impulsividade associadas com o craving (Johnson et al., 2003). Portanto, IFs que reduzem componentes do craving são importantes ferramentas no tratamento da SDA.

\section{Valproato de sódio}

O valproato de sódio foi sintetizado em 1882, contudo sua propriedade anticonvulsivante foi descoberta inesperadamente por Pierrre Eymard (em 1962) quando ele o usou como veículo para dissolver novos compostos que seriam testados para atividade anticonvulsivante. $\mathrm{O}$ valproato de sódio foi aprovado pela FDA para tratamento de enxaqueca, epilepsia e TAB. Na Europa ele tem sido usado para tratar a SAA.

O mecanismo de ação do valproato de sódio consiste no aumento da neurotransmissão GABAérgica, através da intensificação da síntese e liberação do GABA ao atuar nos receptores $\mathrm{GABA}_{\mathrm{A}}$, por meio da ativação da enzima glutâmica descarboxilase e da inibição das enzimas que degradam o GABA (por exemplo, GABA-amino transferase e succinato semi-aldeído desidrogenase). Esse mecanismo é capaz de reduzir a neurotransmissão glutamatérgica por meio do bloqueio dos receptores NMDA, ao inibir a proteinoquinase $\mathrm{C}$ (Zullino et al., 2004). Esse efeito correlaciona-se com a atividade anticonvulsivante do valproato de sódio.

Há pelo menos três principais mecanismos pelos quais se podem aumentar as concentrações do GABA:

- inibição da degradação do GABA;

- aumento da síntese do GABA;

- diminuição do turnover GABAérgico (Owens e Nemeroff, 2003).

Os ensaios clínicos randomizados com valproato de sódio indicam que se trata de uma IF eficaz na redução dos sintomas da SAA, especialmente as convulsões, quando em comparação com placebo, BDZs (lorazepam) e outros anticonvulsivantes (carbamazepina e fenobarbital) nas doses terapêuticas de $1.200 \mathrm{mg} / \mathrm{dia}$, além de níveis séricos em torno de 50 a $100 \mu \mathrm{g} / \mathrm{ml}$. Postula-se que também pode ser eficaz ao reduzir os sintomas de SAP, contudo apresenta a desvantagem das taxas elevadas de abandono em decorrência das altas doses obtidas no início do tratamento, que aumentam sobremaneira a incidência dos efeitos adversos.

Os pacientes muitas vezes necessitam de menores doses de ATCs. A resolução da SAA é mais rápida (Hilbrom et al., 1984; Rosenthal et al., 1998; Myrick et al., 2000; Reoux, 2001; Longo et al., 2002).

Segundo Salloum et al. (2005), o valproato de sódio foi efetivo no tratamento de uma amostra de 59 indivíduos com SDA associada a transtorno bipolar tipo I ao reduzir os dias de consumo pesado de álcool e os níveis séricos de GGT.

É necessário monitoramento cuidadoso da contagem de plaquetas em pacientes em uso de valproato de sódio, especialmente aqueles com abuso de álcool, visto que essa substância, per se, causa trombocitopenia. Recomenda-se também o monitoramento das enzimas hepáticas. Os efeitos adversos mais comuns são náuseas e vômitos. Já as contra-indicações incluem cirrose hepática (com ou sem hipertensão porta) e gravidez. Infelizmente muitos dos ensaios clínicos são abertos, havendo a necessidade de ECRs comparando valproato de sódio com BDZs.

\section{Carbamazepina}

Segundo Malcom et al. (2002), a carbamazepina (CBZ) demonstrou-se superior ao lorazepam ao diminuir o consumo de álcool, isto é, dias de consumo após o término da desintoxicação, principalmente daqueles pacientes com história prévia de múltiplas desintoxicações associadas a alterações do padrão de sono e sintomas de ansiedade, conforme a escala de ansiedade de Zung e a escala analógica visual (Visual Analog Scale [VAS]). A dosagem empregada variou de 600 a $800 \mathrm{mg} / \mathrm{dia}$. 
As taxas de dropout costumam ser elevadas nas doses acima de $800 \mathrm{mg} / \mathrm{dia}$ em decorrência dos seguintes efeitos adversos:

- vertigens;

- náuseas;

- vômitos;

- diplopia;

- rash cutâneo.

Neste ECR com 136 dependentes de álcool, a CBZ e o lorazepam foram igualmente efetivos em diminuir os sintomas da SAA, levando-se em consideração os escores do Clinical Institute Withdrawal Assessment for Alcohol, Revised (CIWA-Ar). Ambas as intervenções farmacológicas não diferiram em relação às taxas de efeitos adversos. $O$ risco de tomar a primeira dose foi três vezes maior no grupo lorazepam que no CBZ. A relação risco/benefício deverá ser avaliada na presença de gravidez, lactação, diabetes mellitus, glaucoma, disfunções hepática e renal, antecedentes de depressão da medula óssea e reações hemáticas adversas por outros medicamentos. O principal mecanismo de ação da CBZ envolve a diminuição da neurotransmissão glutamatérgica através do bloqueio dos receptores NMDA ao inibir o influxo de íons $\mathrm{Ca}^{+2}$ (Zullino et al., 2004).

\section{Gabapentina}

Estudos pré-clínicos sugerem que a gabapentina é eficaz em diminuir as convulsões e os sintomas ansiosos da SAA. É um anticonvulsivante que possui efeitos adversos leves. É considerado um medicamento seguro, pois não há interação com álcool. A posologia recomendada nos primeiros três dias é de $400 \mathrm{mg}$ a cada 8 horas; no quarto dia, a dose é diminuída para $400 \mathrm{mg}$ de $12 \mathrm{em} 12$ horas; e no quinto dia para $400 \mathrm{mg} / \mathrm{dia}$. Além disso, a gabapentina não é metabolizada pelo fígado, tornando-a o anticonvulsivante de escolha para dependentes de álcool com hepatopatias. Ela é excretada de forma inalterada pelos rins, portanto não requer controle laboratorial da dose e/ou de qualquer função do organismo, a não ser a renal (Johnson et al., 2005).

Contudo, há necessidade de mais ECRS para avaliar a eficácia no tratamento da SAA e da SDA. O mecanismo de ação ainda não está totalmente esclarecido. Supõe-se que aumente a neurotransmissão GABAérgica ao promover aumento da síntese e liberação do GABA através da ligação às subunidades dos canais de cálcio tipo $L$, restaurando a ação inibitória do sistema GABAérgico sobre o NA. Postula-se que também exerça ação inibitória sobre o sistema glutamatérgico (Taylor et al., 1998; Johnson et al., 2005).

\section{Referências}

Anton RF, Moak DH, Latham PK. The Obsessive Compulsive Drinking Scale: a new method of assessing outcome in alcoholism treatment studies. Arch Gen Psyhchiatry, 53: 225-31, 1996.

Appolinario JC, Bacaltchuk J. Tratamento farmacológico dos transtornos alimentares. Rev Bras Psiquiatr, 24(Supl III): 54-9, 2002.

Baltieri DA, Andrade AG. Efficacy of acamprosate in the treatment of alcoholdependent outpatients. Rev Bras Psiq, 25: 156-9, 2003.

Becker HC. Kindling in Alcohol Withdrawal. Alcohol Health Res World, 22: 25-32, 1998

Ballenger JC, Post RM. Kindling as a model for alcohol withdrawal
Estudos preliminares evidenciaram a eficácia no tratamento da SAA (Myrick et al., 1998; Bozikas et al., 2002) e da SDA, particularmente os casos de leve a moderada gravidade (Bonnet et al., 1999; Myrick e Anton, 2004).

Karam-Hage e Brower (2003) utilizaram a gabapentina em um estudo clínico aberto para tratar 12 dependentes de álcool que estavam abstinentes há quatro semanas e queixavam-se de insônia. Após oito semanas, o sono havia melhorado significativamente e somente dois dos 12 participantes da pesquisa tiveram recaída. Atualmente estão sendo conduzidos três ECRs patrocinados pelo National Institute on Alcohol Abuse and Alcoholism (NIAAA) com dependentes de álcool para avaliar a sua eficácia na prevenção de recaídas e na redução do consumo de álcool. A gabapentina não deve ser usada durante a gestação nem no período de amamentação.

\section{Conclusão}

Há mais de 30 anos os anticonvulsivantes são investigados no tratamento dos transtornos relacionados ao uso do álcool. Os ATCs representam um campo potencial para estudo de novas IF para tratamento da SDA, pois agem de forma semelhante ao álcool, ou seja, inibem os receptores excitatórios do glutamato e aumentam a neurotransmissão GABAérgica. Em teoria, os ATCs poderiam substituir o álcool na redução dos sintomas da SAP através de sua ação anti-kindling .

Apesar de os ATCs ainda não terem sido aprovados pela FDA, há uma crescente evidência na literatura que sustenta o seu uso, pois, devido à ausência de potencial de abuso, eles representam uma boa alternativa a outras intervenções farmacêuticas (Book e Myrick, 2005).

O topiramato tem múltiplas ações em vários sistemas de neurotransmissores e canais de íons. É uma promissora IF tanto no tratamento da SDA como no da SAA. O TPM, na dose escalonada, que variou de 25 a $300 \mathrm{mg} / \mathrm{dia}$, diminui a freqüência e a quantidade do consumo de álcool quando em comparação com o grupo placebo. É capaz também de reduzir o craving e a impulsividade associada a ele (Johnson et al., 2003; Johnson et al., 2004). Os principais mecanismos de ação do TPM envolvem a potencialização da atividade GABAérgica e o antagonismo dos receptores AMPA/cainato.

A carbamazepina, a gabapentina e o valproato de sódio teriam uma indicação restrita ao tratamento coadjuvante da SAA. É importante ressaltar que há necessidade de mais ECRs com a CBZ e o valproato de sódio para uma ampla utilização terapêutica na SDA.

episodes and the severity of subsequent withdrawal seizures. Psychopharmacology, 116: 26-32, 1978.

Bonnet U, Banger M, Leweke FM et al. Treatment of alcohol withdrawal syndrome with gabapentin. Pharmacopsychiatry, 32: 107-9, 1999.

Book SW, Myrick H. Novel anticonvulsants in the treatment of alcoholism. Expert Opin Investig Drugs, 14(4): 371-6, 2005.

Bozikas V, Petrikis P, Gamvrula K, Savvidou I, Karavatos A. Treatment of alcohol withdrawal with gabapentin. Prog Neuro-Psychoph Biol Psychiatry, 26: 197-9, 2002. 
Carlini EA, Galduróz JCF, Noto AR, Nappo SA. Levantamento domiciliar sobre 0 uso de drogas psicotrópicas no Brasil: estudo envolvendo as 107 maiores cidades do país - 2001. São Paulo: Cromosete Gráfica e Editora; 2002.

Castro LA, Baltieri DA. The pharmacologic treatment of the alcohol dependence. Rev Bras Psiq, 26(Suppl 1): S43-6, 2004.

Carrol KM, Nich C, Ball SA, McCance E, Rounsavile BJ. Treatment of cocaine and alcohol dependence with psychotherapy and disulfiram. Addiction, 93: 713-27, 1998.

Chick J, Gough K, Falkowski W, Kershaw P, Hore B, Mehta B, Ritson B, Ropner R, Torley D. Disulfiram treatment of alcoholism. Br J Psychiatry, 161: 84-9, 1992.

Di Chiara G. Alcohol and dopamine. Alcohol Health Res World, 21: 108-13, 1997.

Fuller RK, Branchey L, Brightwell DR, Derman RM, Emrick CD, Iber FL, James KE, Lacoursiere RB, Lee KK, Lowenstam L. Dissulfiram treatment of alcoholism: a Veteran's Administration Cooperative Study. JAMA, 256: 1449-55, 1996.

Fuller RK, Gordis E. Doe's disulfiram have a role in alcoholism treatment today? Addicition, 99: 21-4, 2004.

Garbutt JC, West SL, Carey TS, Lohr KN, Crews FT. Pharmacological treatment of alcohol dependence: a review of the evidence. JAMA 281: 1318-25, 1999.

Goddard GV, Mclntyre, DC, Lech DC. A permanent change in brain function resulting from daily electrical stimulation. Exp Neurol, 25: 295-330, 1969.

Gonzales RA, Jaworski JN. Alcohol and glutamate. Alcohol Health Res World, 21(2): 120-7, 1997

Hartz DT, Frederick-Osborne SL, Galloway GP. Craving predicts use during treatment for methamphetamine dependence: a prospective, repeated-measures, within-subject analysis. Drug Alcohol Depend, 63(3): 269-76, 2001.

Hillbrom ME, Hjelm-Jager M. Should alcohol withdrawal seizures be treated with anti-epileptic drugs? Acta Neurol Scand, 69: 39-42, 1984.

Karam-Hage M, Brower KJ. Open pilot study of gabapentin versus trazodone to treat insomnia in alcoholic outpatients. Psychiatry Clin Neurosci, 57(5): 542-4, 2003.

Knopfel T, Zeise ML, Cuenod M, Zieglgansberger W. L-homocisteric acid but not I-glutamate is an endogenous N-metil-D-aspartic acid receptor preferin agonist in rat neocortical neurons in vitro. Neurocience, 81 188-92, 1987

Johnson BA, Ait-Daoud N, Bowden CL, DiClement CC, Roache JD, Lawson K, Javors MA, Ma JZ. Oral topiramato for treatment of alcohol dependence: a randomized controlled trial. Lancet, 361: 1677-85, 2003.

Johnson BA, Swift RM, Ait-Daoud N, DiClemente CC, Martin AJ, Robert JMJ. Development of novel pharmacotherapies for the treatment of alcohol dependence: focus on antiepileptics. Alcohol Clin Exp Res, 28: 295-301, 2004

Johnson BA, Swift RM, Addolorato G, Ciraulo DA, Myrick H. Safety and efficacy of GABAergic medications for treating alcoholism. Alcohol Clin Exp Res, 29(2): 248-54, 2005.

Laranjeira R, Nicastri S, Jerônimo C, Marques AC et al. Consenso sobre a síndrome de abstinência do álcool (SAA) e o seu tratamento. Rev Bas Psiq, 22(2): 62-71, 2000

Lechtenberg R, Worner TM. Relative kindling effect of detoxification and non-detoxification admissions in alcoholics. Alcohol Alcoholism, 26(2) 221-5, 1991.

Litleton, J. Acamprosate in alcohol dependence. How does work? Addiction, 90: 1179-88, 1995.

Litten RZ, Allen J, Fertig J. Pharmacoterapies for alcohol problems: a review of research with focus on developments since 1991. Alcohol Clin Exp Res, 20: 859-76, 1996.

Litten RZ, Fertig J, Mattson ME, Egli M. Development of medications for alcohol use disorders: recent advances and ongoing challenges. Expert Opin Emergind Drugs, 10(2): 323-43, 2005
Longo LP, Campbell C, Hubatch S. Divalproex sodium for alcohol withdrawal and relapse prevention. J Addict Dis, 21: 55-64, 2002.

Malcolm R, Myrick H, Brady KT, Ballenger JC. Update on anticonvulsants for the treatment os alcohol withdrawal. Am J Addict, 10(Suppl): 1623, 2001.

Malcolm R, Myrick H, Roberts J, Wang W, Anton RF, Malcolm R, Myrick H, Roberts $\mathrm{J}$ et al. The differential effects of medication on psychiatric and psychosocial outcomes in outpatient alcohol detoxification. Am J Addict, 11(2): 141-50, 2002.

McNamara JO, Wada JA. Kindling model. In: Engel J, Pedley TA, editors Epilepsy: a Comprehensive Texbook. Philadelphia: Lippincott-Raven Publishers; 1997.

Mihic SJ, Harris RA. GABA and the GABA receptor. Alcohol Res Health World, 21: 127-32, 1997.

Moak DH, Anton RF. Alcohol-related seizures and the kindling effect of repeated detoxifications: the influence of cocaine. Alcohol Alcoholism, 31: 135-43, 1996.

Monterosso JR, Flannery BA, Pettinati HM, Oslin DW, Ruktalis M, O'Brien $\mathrm{CP}$, Volpicelli JR. Predicting treatment response to naltrexone: the influence of craving and family history. Am J Addict, 10(3): 258-68, 2001.

Moreno RA, Moreno DH, Soares MBM, Ratske R. Anticonvulsivantes e antipsicóticos no tratamento do transtorno bipolar. Rev Bras Psiq, 26(Supl III): 37-43, 2004.

Myrick H, Malcolm R, Brady KT. Gabapentin treatment of alcohol withdrawal. Am J Psychiatry, 155: 1632, 1998.

Myrick H, Brady KT, Malcolm R. Divalproex in the treatment of alcohol withdrawal. Am J Drug Alcohol Abuse, 26: 155-60, 2000.

Myrick $\mathrm{H}$, Anton R. Recent advances in the pharmacotherapy of alcoholism Curr Psychiatry Rep, 6: 332-8, 2004.

Nalpas B, Dabadie H, Parot P, Paccalin J. L'amprosate: de la pharmocologie a la clinique. Encephale, 16: 175-9, 1990.

O'Malley SS, Jaffe A, Chang G, Schottenfeld RS, Meyer RE, Rounsaville B. Naltrexone and coping sills therapy for alcohol dependence: a controlled study. Arch Gen Psychiatry, 49: 881-7, 1992.

Owens MJ, Nemeroff CB. Pharmacology of valproate. Psychoph Bull, 37 17-24, 2003

Reoux JP, Saxon AJ, Malte CA, Baer JS, Sloan KL. Divalproex in alcohol withdrawal: a randomized double-blind placebo-controlled clinical trial. Alcohol Clin Exp Res, 25: 1324-9, 2001.

Rosenthal RN, Perkel C, Singh P, Anand O, Miner CR. A pilot trial of valproate and phenobarbital in the treatment of alcohol withdrawal. Am J Addiction, 7: 189-97, 1998.

Salloum IM, Cornelius JR, Daley DC, Kirisci L, Himmelhoch JM, Thase ME. Efficacy of valproate maintenance in patients with bipolar disorder and alcoholism: a double-blind placebo controlled study. Arch Gen Psychiatry, 62: 37-45, 2005.

Spanagel R, Holter SM, Allingham K, Landgraf R, Zieglgansberger W. Acamprosate and alcohol: effects on alcohol intake following alcohol deprivation in the rat. Eur J Pharmacol, 305: 39-44, 1996.

Swift RM. Topiramate for the treatment of alcohol dependence: initiating abstinence. Lancet, 361: 1666-7, 2003.

Taylor CP, Gee NS, Su T-Z, Kocsis JD, Welty DF, Brown JP, Dooley DJ, Boden P, Singh L. A summary of mechanistic hypothesis of gabapentin pharmachology. Epilepsy Res, 29(3): 231-46, 1998.

Volpicelli JR, Alterman AL, Hayashida, M, O'Brien CP. Naltrexone in the treatment of alcohol dependence. Arch Gen Psychiatry, 49: 876-80, 1992.

Zeise ML, Kasparov S, Capogna M, Zieglgansberger W. Acamprosate (calcium homoturinate) decrease post-sinaptic potentials in the rat neicotex: possible involvement of excitatory amino acid receptors Eur J Pharmacol, 231: 47-52, 1993.

Zullino DF, Khazaal Y, Hattenschwiler J, Borgeat F, Besson J. Anticonvulsant drugs in the treatment of substance withdrawal. Drugs Today, 40(7): 603-19, 2004 